DOI: $10.36910 / 6775-2524-0560-2019-37-9$

УДК: 539.3

Грінченко Л. Г.

Луцький національний технічний університет

\title{
МЕТОД КОЛЛОКАЦІЙ СИСТЕМ ЛІНІЙНИХ РІВНЯНЬ ЗІ ЗМІННИМИ КОЕФІЦІЕТАМИ
}

Грінченко Л.Г. Метод коллокацій систем лінійних рівнянь зі змінними коефіцієнтами. В роботі розглянутий згин тонкої ізотропної прямокутної плити , з певними граничними умовами Через базові функції і функції форми визначені: функція прогину, кути поворотів нормалей. Проведено числові розрахунки розподілу, прогину та згинних моментів в головних перерізах плити.

Ключові слова: плита, напруження, згин.

Гринченко Л.Г. Метод коллокации систем линейных уравнений с переменными коэффициентами. В работе рассмотрен изгиб тонкой изотропной прямоугольной плиты, с определенными граничными условиями. Через базовые функции и функции формы определены: функция прогиба, углы поворота нормалей Проведены числовые расчеты для распределения прогиба и сгинальных моментов в главных разрезах плиты.

Ключевые слова: плита, напряжение, изгиб.

Grinchenko L.G. Collocation method of systems of linear equations with variable koefficients. In the work is studied the bend of thin isotop rectangular plate, with definite limiting conditions. Through the basic functions and the functions of the form are determined function of deflexion and angles of normal bending. Numerical calculations of distribution, bending and curving moments in main cutting of the plate.

Keywords: flag, tension, bend.

Розглянемо тонку прямокутну ізотропну плиту з розмірами в плані $2 a_{\mathrm{j}}(j=1,2)$ і товщиною $h$ жорстко закріплену на контурі і завантажену на верхній поверхні сталим навантаженням $q\left(x_{1}, x_{2}\right)=q_{0}$. Згин такої пластини описується диференціальним рівнянням четвертого порядку [1-4]:

$$
\frac{\partial^{4} w}{\partial x_{1}^{4}}+2 \frac{\partial^{4} w}{\partial x_{1}^{2} \partial x_{2}^{2}}+\frac{\partial^{4} w}{\partial x_{2}^{4}}=\frac{q_{0}}{D}
$$

Тут $w\left(x_{1}, x_{2}\right)$ - прогин плити; $D=\frac{E h^{3}}{12\left(1-v^{2}\right)}$ - iї циліндрична жорсткість; де $E$ і $v$ відповідно модуль Юнга і коефіцієнт Пуасона матеріалу плити. Моменти $M_{11}, M_{22}$ в плиті визначаються за формулами: $M_{11}=-D\left(\frac{\partial^{2} w}{\partial x_{1}^{2}}+v \frac{\partial^{2} w}{\partial x_{2}^{2}}\right)$,

$$
M_{22}=-D\left(\frac{\partial^{2} w}{\partial x_{2}^{2}}+v \frac{\partial^{2} w}{\partial x_{1}^{2}}\right)
$$

через функцію прогину $w\left(x_{1}, x_{2}\right)$.Для розрахунку напружено-деформованого стану плити необхідно знати функцію прогину $w\left(x_{1}, x_{2}\right)$. Знаходимо iï $з$ розв'язку диференціального рівняння (1), задовільнивши при цьому відповідні граничні умови.Загальний розв'язок неоднорідного диференціального рівняння (1) шукаємо у вигляді суми

$$
w=w_{0}+w_{*}
$$

загального розв'язку $w_{0}$ однорідного диференціального рівняння: $\frac{\partial^{4} w}{\partial x_{1}^{4}}+2 \frac{\partial^{4} w}{\partial x_{1}^{2} \partial x_{2}^{2}}+\frac{\partial^{4} w}{\partial x_{2}^{4}}=0$

і часткового розв'язку $\mathcal{W}_{*}$ неоднорідного диференціального рівняння (1). Цей розв'язок вибираємо у вигляді:

$$
w_{*}=\frac{q_{0}\left(x_{1}^{2}-a_{1}^{2}\right) \cdot\left(x_{2}^{2}-a_{2}^{2}\right)}{8 D} .
$$

$w_{0}=\sum_{k=1}^{\text {Загальний }}\left[f_{k}^{[1]}\left(x_{1}\right) \cos \delta_{k}^{[2]} x_{2}+f_{k}^{[2]}\left(x_{2}\right) \cos \delta_{k}^{[1]} x_{1}\right]$

шукаємо у вигляді

Підставивши вираз (6) до рівняння (4) після розділення змінних приходимо до системи двох незв'язних диференціальних рівнянь $f_{k}^{[j]^{(I V)}}\left(x_{j}\right)-2 \delta_{k}^{[3-j]^{(2)}} f_{k}^{[j]^{\prime \prime}}\left(x_{j}\right)+\delta_{k}^{[3-j]^{4}} f_{k}^{[j]}\left(x_{j}\right)=0, j=1,2$

на невідомі функції $f_{k}^{[j]}\left(x_{j}\right)$. Часткові розв'язки даної системи вибираємо у вигляді $f_{k}^{[j]}\left(x_{j}\right)=R_{k}^{[j] *} \exp \left(\lambda_{k}^{[j]} x_{j}\right)$, де $R_{k}^{[j]^{*}}, \lambda_{k}^{[j]}$ невідомі параметри. Підставляючи ці розв'язки до рівнянь (7) отримуємо характеристичні рівняння на параметри $\lambda_{k}^{[j]}: \lambda_{k}^{[j]^{4}}-2 \delta_{k}^{[3-j]^{2}} \cdot \lambda_{k}^{[j]^{2}}+\delta_{k}^{[3-j]^{4}}=0$.

$$
\text { Корені } \quad \lambda_{(1,2) k}^{[j]}=\delta_{k}^{[3-j]} ; \quad \lambda_{(3,4) k}^{[j]}=-\delta_{k}^{[3-j]}
$$


цих рівнянь є кратні, тому загальний розв'язок однорідного рівняння (4) набуває вигляду:

$$
\begin{aligned}
& w_{0}=\sum_{k=1}^{\infty}\left\{\left[R_{1(k)}^{[1]^{*}} \exp \left(\delta_{k}^{[2]} x_{1}\right)+R_{2(k)}^{[1]^{*}} x_{1} \exp \left(\delta_{k}^{[2]} x_{1}\right)\right] \cos \left(\delta_{k}^{[2]} x_{2}\right)+\right. \\
& \left.+\left[R_{1(k)}^{[2]^{*}} \exp \left(\delta_{k}^{[1]} x_{2}\right)+R_{2(k)}^{[2]^{*}} x_{2} \exp \left(\delta_{k}^{[1]} x_{2}\right)\right] \cos \left(\delta_{k}^{[1]} x_{1}\right)\right\}
\end{aligned}
$$

Оскільки розглядувана задача $є$ симетричною то прогин плити повинен бути парною функцією змінних змінних $x_{1}$, $x_{2}$. Представимо його у вигляді:

$$
\begin{aligned}
& w_{0}=\sum_{k=1}^{\infty}\left\{\left[R_{1(k)}^{[1]^{*}} \operatorname{ch}\left(\delta_{k}^{[2]} x_{1}\right)+R_{2(k)}^{[1]^{*}} x_{1} \operatorname{sh}\left(\delta_{k}^{[2]} x_{1}\right)\right] \cos \left(\delta_{k}^{[2]} x_{2}\right)+\right. \\
& \left.+\left[R_{1(k)}^{[2]^{*}} \operatorname{ch}\left(\delta_{k}^{[1]} x_{2}\right)+R_{2(k)}^{[2]^{*}} x_{2} \operatorname{sh}\left(\delta_{k}^{[1]} x_{2}\right)\right] \cos \left(\delta_{k}^{[1]} x_{1}\right)\right\}
\end{aligned}
$$

При збільшенні кількості наближень $k$ експоненціальні функції суттєво зростають. Тому для кращої можливості проведення числових розрахунків розв'язок (12), нормуємо представивши його у вигляді [6]:

де

$$
\begin{aligned}
& w_{0}=\sum_{k=1}^{\infty}\left\{\left[\tilde{R}_{1(k)}^{[1]} \Phi_{1(k)}^{[1]}\left(x_{1}\right)+\tilde{R}_{2(k)}^{[1]} \Phi_{2(k)}^{[1]}\left(x_{1}\right)\right] C_{k}^{[2]}\left(x_{2}\right)+\right. \\
& \left.+\left[\tilde{R}_{1(k)}^{[2]} \Phi_{1(k)}^{[2]}\left(x_{2}\right)+\tilde{R}_{2(k)}^{[2]} \cdot \Phi_{1(k)}^{[2]}\left(x_{2}\right)\right] \cdot C_{k}^{[1]}\left(x_{1}\right)\right\},
\end{aligned}
$$

$$
\begin{gathered}
\Phi_{1(k)}^{[j]}\left(x_{j}\right)=\frac{\operatorname{ch}\left(\delta_{k}^{[3-j]} x_{j}\right)}{\exp \left(\delta_{k}^{[3-j]} a_{j}\right)} ; \quad \Phi_{2(k)}^{[j]}=\frac{x_{j}}{a_{j}} \cdot \frac{\operatorname{sh}\left(\delta_{k}^{[3-j]} x_{j}\right)}{\exp \left(\delta_{k}^{[3-j]} a_{j}\right)} \\
\widetilde{R}_{1(k)}^{[j]}=R_{1(k)}^{[j]^{*}} \exp \left(\delta_{k}^{[3-j]} a_{j}\right) ; \widetilde{R}_{2(k)}^{[j]}=R_{2(k)}^{[j]^{*}} \cdot a_{j} \exp \left(\delta_{k}^{[3-j]} a_{j}\right) ; \\
C_{k}^{[j]}\left(x_{j}\right)=\cos \left(\delta_{k}^{[3-j]} x_{j}\right) ; \quad \delta_{k}^{[j]}=\frac{(2 k-1) \pi}{2 a_{j}} ; \quad \delta_{k}=\frac{(2 k-1) \pi}{2} .
\end{gathered}
$$

Загальний розв'язок неоднорідного рівняння (1) отримуємо за формулою (3), враховуючи співвідношення (5), (13). Подамо його в безрозмірних величинах

$$
\begin{aligned}
& w=\frac{q_{0} a_{1}^{4}}{D} \sum_{k=1}^{\infty}\left\{\left[\tilde{R}_{1(k)}^{[1]} \Phi_{1(k)}^{[1]}\left(x_{1}\right)+\tilde{R}_{2(k)}^{[1]} \Phi_{2(k)}^{[1]}\left(x_{1}\right)\right] C_{k}^{[2]}\left(x_{2}\right)+\right. \\
& \left.+\left[\tilde{R}_{1(k)}^{[2]} \Phi_{1(k)}^{[2]}\left(x_{2}\right)+\tilde{R}_{2(k)}^{[2]} \cdot \Phi_{1(k)}^{[2]}\left(x_{2}\right)\right] C_{k}^{[1]}\left(x_{1}\right)+\frac{\left(x_{1}^{2} / a_{1}^{2}-1\right)\left(x_{2}^{2} / a_{2}^{2}-1\right)}{8}\right\} .
\end{aligned}
$$

Диференціюючи функцію прогину $w\left(x_{1}, x_{2}\right)$ по змінних $x_{1}, x_{2}$ отримуємо вирази на кути поворотів нормалей до серединної поверхні плити після деформації

$$
\begin{gathered}
\varphi_{1}=\frac{\partial w}{\partial x_{1}}=\frac{q_{0} a_{1}^{4}}{D} \sum_{k=1}^{\infty}\left\{\left[\tilde{R}_{1(k)}^{[1]} \Phi_{1(k)}^{[1]}{ }^{\prime}\left(x_{1}\right)+\tilde{R}_{2(k)}^{[1]} \Phi_{2(k)}^{[1]}{ }^{\prime}\left(x_{1}\right)\right] C_{k}^{[2]}\left(x_{2}\right)+\right. \\
\left.+\left[\tilde{R}_{1(k)}^{[2]} \Phi_{1(k)}^{[2]}\left(x_{2}\right)+\tilde{R}_{2(k)}^{[2]} \cdot \Phi_{1(k)}^{[2]}\left(x_{2}\right)\right] C_{k}^{[1]}\left(x_{1}\right)+\frac{x_{1}\left(x_{2}^{2} / a_{2}^{2}-1\right)}{4}\right\} . \\
\varphi_{2}=\frac{\partial w}{\partial x_{2}}=\frac{q_{0} a_{1}^{4}}{D} \sum_{k=1}^{\infty}\left\{\left[\tilde{R}_{1(k)}^{[1]} \Phi_{1(k)}^{[1]}\left(x_{1}\right)+\tilde{R}_{2(k)}^{[1]} \Phi_{2(k)}^{[1]}\left(x_{1}\right)\right] C_{k}^{[2]}\left(x_{2}\right)+\right. \\
\left.+\left[\tilde{R}_{1(k)}^{[2]} \Phi_{1(k)}^{[2]}\left(x_{2}\right)+\tilde{R}_{2(k)}^{[2]} \cdot \Phi_{1(k)}^{[2]}{ }^{\prime}\left(x_{2}\right)\right] C_{k}^{[1]}\left(x_{1}\right)+\frac{x_{2}\left(x_{1}^{2} / a_{1}^{2}-1\right) a_{2}^{2}}{4 a_{1}^{2}}\right\} .
\end{gathered}
$$

Невідомі сталі $x_{j}= \pm a_{j}$ маємо граничні умови: $\left.w\right|_{x_{i}=a_{i}}=0 ; \quad \varphi_{i}=\left.\frac{\partial w}{\partial x_{i}}\right|_{x_{i}=a_{i}}=0$;

де $\varphi_{i}\left(x_{i}\right)$ кут повороту грані $x_{i}=a_{i}$.

Для зручності обчислень запишемо отримані співвідношення в безрозмірних змінних

$$
\left.\xi_{j}=x_{j} / a_{j}\left(-1 \leq \xi_{j} \leq 1\right) \quad+\left[R_{1(k)}^{[2]} \cdot \Phi_{1(k)}^{[2]}\left(\xi_{2}\right)+R_{2(k)}^{[2]} \cdot \Phi_{2(k)}^{[2]}\left(\xi_{2}\right)\right] \cdot C_{k}^{[1]}\left(\xi_{1}\right)\right\}+\frac{q_{0} \cdot a_{2}^{2}\left(\xi_{1}^{2}-1\right)\left(\xi_{2}^{2}-1\right)}{8 a_{1}^{2}}
$$




$$
\begin{aligned}
& \frac{\partial w}{\partial x_{1}}=\left(\frac{q_{0} a_{1}^{4}}{D}\right)\left[\frac { 1 } { a _ { 1 } } \sum _ { k = 1 } ^ { K } \left\{\left[\widetilde{R}_{1(k)}^{[1]} \cdot \Phi_{1(k)}^{[1]}{ }^{\prime}\left(\xi_{1}\right)+\widetilde{R}_{2(k)}^{[1]} \cdot \Phi_{2(k)}^{[1]}{ }^{\prime}\left(\xi_{1}\right)\right] \cdot C_{k}^{[2]}\left(\xi_{2}\right)+\right.\right. \\
& \left.\left.+\left[\widetilde{R}_{1(k)}^{[2]} \cdot \Phi_{1(k)}^{[2]}\left(\xi_{2}\right)+\widetilde{R}_{2(k)}^{[2]} \cdot \Phi_{2(k)}^{[2]}\left(\xi_{2}\right)\right] \cdot C_{k}^{[1]^{\prime}}\left(\xi_{1}\right)\right\}+\frac{a_{2}^{2}}{8 a_{1}^{2}}\left(\xi_{2}^{2}-1\right) 2 \xi_{1} \cdot \frac{1}{a_{1}}\right] ; \\
& \frac{\partial w}{\partial x_{2}}=\left(\frac{q_{0} a_{1}^{4}}{D}\right)\left[\frac { 1 } { a _ { 2 } } \sum _ { k = 1 } ^ { K } \left\{\left[\widetilde{R}_{1(k)}^{[1]} \cdot \Phi_{1(k)}^{[1]}\left(\xi_{1}\right)+\widetilde{R}_{2(k)}^{[1]} \cdot \Phi_{2(k)}^{[1]}\left(\xi_{1}\right)\right] \cdot C_{k}^{[2]}\left(\xi_{2}\right)+\right.\right. \\
& \left.\left.+\left[\widetilde{R}_{1(k)}^{[2]} \cdot \Phi_{1(k)}^{[2]}{ }^{\prime}\left(\xi_{2}\right)+\widetilde{R}_{2(k)}^{[2]} \cdot \Phi_{2(k)}^{[2]}{ }^{\prime}\left(\xi_{2}\right)\right] \cdot C_{k}^{[1]}\left(\xi_{1}\right)\right\}+\frac{a_{2}^{2}}{8 a_{1}^{2}}\left(\xi_{1}^{2}-1\right) 2 \xi_{2} \cdot \frac{1}{a_{2}}\right] \text {; } \\
& \text { де: } \quad R_{1(k)}^{[j]}=\frac{\tilde{R}_{1(k)}^{[j]}}{\left(\frac{q_{0} a_{1}^{4}}{D}\right)} ; \quad R_{2(k)}^{[j]}=\frac{\tilde{R}_{2(k)}^{[j]}}{\left(\frac{q_{0} a_{1}^{4}}{D}\right)} ; \\
& \Phi_{1(k)}^{[j]}\left(\xi_{j}\right)=\frac{1}{2} \exp \left[-\delta_{k} \cdot \frac{a_{j}}{a_{3-j}}\left(1-\xi_{j}\right)\right]+\frac{1}{2} \exp \left[-\delta_{k} \cdot \frac{a_{j}}{a_{3-j}} \cdot\left(1+\xi_{j}\right)\right] \text {; } \\
& \Phi_{2(k)}^{[j]}\left(\xi_{j}\right)=\frac{1}{2} \xi_{j} \exp \left[-\delta_{k} \cdot \frac{a_{j}}{a_{3-j}}\left(1-\xi_{j}\right)\right]-\frac{1}{2} \cdot \xi_{j} \cdot \exp \left[-\delta_{k} \cdot \frac{a_{j}}{a_{3-j}} \cdot\left(1+\xi_{j}\right)\right] ; \\
& \Phi_{1(k)}^{[j]^{\prime}}\left(\xi_{j}\right)=\frac{1}{2} \exp \left[-\delta_{k} \cdot \frac{a_{j}}{a_{3-j}}\left(1-\xi_{1}\right)\right] \cdot \delta_{k} \frac{a_{j}}{a_{3-j}}-\frac{1}{2} \exp \left[-\delta_{k} \cdot \frac{a_{j}}{a_{3-j}}\left(1+\xi_{j}\right)\right] \delta_{k} \cdot \frac{a_{j}}{a_{3-j}} ; \\
& \Phi_{2(k)}^{[j]^{\prime}}\left(\xi_{j}\right)=\frac{1}{2} \exp \left[-\delta_{k} \cdot \frac{a_{j}}{a_{3-j}}\left(1-\xi_{j}\right)\right]+\frac{1}{2} \xi_{2} \cdot \exp \left[-\delta_{k} \cdot \frac{a_{j}}{a_{3-j}}\left(1-\xi_{j 2}\right)\right] \delta_{k} \frac{a_{j}}{a_{3-j}}- \\
& -\frac{1}{2} \exp \left[-\delta_{k} \cdot \frac{a_{2}}{a_{1}}\left(1+\xi_{2}\right)\right]+\frac{1}{2} \xi_{2} \exp \left[-\delta_{k} \cdot \frac{a_{2}}{a_{1}}\left(1+\xi_{2}\right)\right] \cdot \delta_{k} \cdot \frac{a_{2}}{a_{1}} ; \\
& \text { Підставлюючи вирази (17), (18), (20) до граничних умов } w_{\xi_{1}=1}=0 ; \quad \varphi_{1}=\left.\frac{\partial w}{\partial x_{1}}\right|_{\xi_{1}=1}=0 \text {; } \\
& \left.w\right|_{\xi_{2}=1}=0 ; \quad \varphi_{2}=\left.\frac{\partial w}{\partial x_{2}}\right|_{\xi_{2}=1}=0 ;
\end{aligned}
$$

і враховуючи, що $C_{k}^{[1]}(1)=C_{k}^{[2]}(1)=0$ отримуємо систему $2 k+2$ алгебраїчних рівнянь на невідомі коефіцієнти $\widetilde{R}_{v(k)}^{[j]}$,

$\begin{array}{ll} & \widetilde{R}_{1(k)}^{[1]} \cdot \Phi_{1(k)}^{[1]}(1)+\widetilde{R}_{2(k)}^{[1]} \cdot \Phi_{2(k)}^{[1]}(1)=0 ; \\ & \widetilde{R}_{1(k)}^{[2]} \cdot \Phi_{1(k)}^{[2]}(1)+\widetilde{R}_{2(k)}^{[2]} \cdot \Phi_{2(k)}^{[2]}(1)=0 ;\end{array}$

$$
\begin{aligned}
& \sum_{k=1}^{K}\left\{\left[\tilde{R}_{1(k)}^{[1]} \cdot \Phi_{1(k)}^{[1]}{ }^{\prime}(1)+\widetilde{R}_{2(k)}^{[1]} \cdot \Phi_{2(k)}^{[1]}{ }^{\prime}(1)\right] \cdot C_{k}^{[2]}\left(\xi_{2}\right)+\right. \\
& \left.+\left[\tilde{R}_{1(k)}^{[2]} \cdot \Phi_{1(k)}^{[2]}\left(\xi_{2}\right)+\tilde{R}_{2(k)}^{[2]} \cdot \Phi_{2(k)}^{[2]}\left(\xi_{2}\right)\right] \cdot C_{k}^{[1] '}(1)\right\}+\frac{a_{2}^{2}}{4 a_{1}^{2}}\left(\xi_{2}^{2}-1\right)=0 \\
& \quad \sum_{k=1}^{K}\left\{\left[\tilde{R}_{1(k)}^{[1]} \cdot \Phi_{1(k)}^{[1]}\left(\xi_{1}\right)+\tilde{R}_{2(k)}^{[1]} \cdot \Phi_{2(k)}^{[1]}\left(\xi_{1}\right)\right] \cdot C_{k}^{[2]}{ }^{\prime}(1)+\right. \\
& \left.\quad+\left[\tilde{R}_{1(k)}^{[2]} \cdot \Phi_{1(k)}^{[2]}(1)+\tilde{R}_{2(k)}^{[2]} \cdot \Phi_{2(k)}^{[2]}{ }^{\prime}(1)\right] \cdot C_{k}^{[1]}\left(\xi_{1}\right)\right\}+\frac{a_{2}^{2}}{4 a_{1}^{2}}\left(\xi_{1}^{2}-1\right)=0 ;
\end{aligned}
$$

3 перших $2 k$ рівнянь системи (24) знаходимо:

$$
R_{2(k)}^{[j]}=-\frac{\Phi_{1(k)}^{[j]}(1)}{\Phi_{2(k)}^{[j]}(1)} R_{1(k)}^{[j]} \quad ;
$$

Підставляючи ці зв'язки до інших двох рівнянь даної системи отримуємо систему двох рівнянь зі змінними коефіцієнтами на визначення невідомих коефіцієнтів $R_{1(k)}^{[1]}, R_{2(k)}^{[2]}$ 


$$
\begin{aligned}
& \quad \sum_{k=1}^{K}\left\{R_{1(k)}^{[1]}\left[\Phi_{1(k)}^{[1]}{ }^{\prime}(1)-\frac{\Phi_{1(k)}^{[1]}(1)}{\Phi_{2(k)}^{[1]}(1)} \Phi_{2(k)}^{[1]}{ }^{\prime}(1)\right] \cdot C_{k}^{[2]}\left(\xi_{2}\right)+\right. \\
& \left.\quad+R_{1(k)}^{[2]}\left[\Phi_{1(k)}^{[2]}\left(\xi_{2}\right)-\frac{\Phi_{1(k)}^{[2]}(1)}{\Phi_{2(k)}^{[2]}(1)} \Phi_{2(k)}^{[2]}\left(\xi_{2}\right)\right] \cdot C_{k}^{[1] \prime}(1)\right\}+\frac{a_{2}^{2}}{4 a_{1}^{2}}\left(\xi_{2}^{2}-1\right)=0 \\
& \sum_{k=1}^{K}\left\{R_{1(k)}^{[1]}\left[\Phi_{1(k)}^{[1]}\left(\xi_{1}\right)-\frac{\Phi_{1(k)}^{[1]}(1)}{\Phi_{2(k)}^{[1]}(1)} \Phi_{2(k)}^{[1]}\left(\xi_{1}\right)\right] \cdot C_{k}^{[2]}(1)+\right. \\
& \left.+R_{1(k)}^{[2]}\left[\Phi_{1(k)}^{[2]}(1)-\frac{\Phi_{1(k)}^{[1]}(1)}{\Phi_{2(k)}^{[1]}(1)} \cdot \Phi_{2(k)}^{[2]}(1)\right] \cdot C_{k}^{[1]}\left(\xi_{1}\right)\right\}+\frac{a_{2}^{2}}{4 a_{1}^{2}}\left(\xi_{1}^{2}-1\right)=0 ;
\end{aligned}
$$

Систему рівнянь (26), (27) розв'язуємо методом колокацій. Для цього проміжок $\xi_{1} \in[0 ; 1]$ та $\xi_{2} \in[0 ; 1]$ розбиваємо на ряд інтервалів довжиною $\frac{1}{K}$ і записуємо цю систему рівнянь для кожної точки: $\xi_{1}=0 ; \xi_{1}=\frac{1}{K} ; \xi_{1}=\frac{2}{K} ; \ldots, \xi_{1}=\frac{K-1}{K}$ та $\xi_{2}=0 ; \xi_{2}=\frac{1}{K} ; \xi_{2}=\frac{2}{K} ; \ldots, \xi_{2}=\frac{K-1}{K}$.

Кількість точок колокацій (параметр К) вибираємо так, щоб досягти заданої точності задоволення системи рівнянь (26), (27). В рахунках покладено $v=0,3$. Точність задоволення граничних умов перевіряли за максимальним відхиленням від нуля лівих частин рівнянь (26), (27 при відомих уже коефіцієнтах $R_{v(k)}^{[j]}$, табулюючи їх як функції від $\xi_{1}, \xi_{2}$ з кроком $H=0.01$. В результаті табуляції встановлено, що для десяти точок колокацій $(K=10)$ максимальне відхилення від нуля за абсолютною величиною на всьому інтервалі $\xi_{j} \in[-1 ; 1]$ у випадку, коли відношення сторін плити задовільняє умові: $\frac{a_{1}}{a_{2}}=0.5 ; 0.6 ; 0.7 ; 0.8 ; 0.9 ; 1.0$ для рівняння (26) не перевищує $9.551644 \cdot 10^{-5}$, для рівняння (27) не перевищує 4.864484.10-5. У випадку відношення $\frac{a_{1}}{a_{2}}=1.11 ; 1.25 ; 1.43 ; 1.67 ; 2.00$ максимальне відхилення від нуля за абсолютною величиною для рівняння (26) не перевищує $4.856289 \cdot 10^{-5}$, для рівняння (27) не перевищує $9.549409 \cdot 10^{-5}$, тобто точність задоволення граничних умов $\epsilon$ достатньо висока. Знаючи параметри $R_{v(k)}^{[j]}$ визначаємо прогин $w\left(x_{1}, x_{2}\right)$ в кожній точці плити, а маючи функцію прогину за формулами (2) легко знаходимо моменти в плиті. Запишемо вирази для кривин волокон через які визначаються плиті $\frac{\partial^{2} w}{\partial x_{1}^{2}}=\left(\frac{q_{0} a_{1}^{4}}{D}\right)\left[\frac{1}{a_{1}^{2}} \sum_{k=1}^{K}\left\{\left[\widetilde{R}_{1(k)}^{[1]} \cdot \Phi_{1(k)}^{[1]} "\left(\xi_{1}\right)+\widetilde{R}_{2(k)}^{[1]} \cdot \Phi_{2(k)}^{[1]} "\left(\xi_{1}\right)\right] \cdot C_{k}^{[2]}\left(\xi_{2}\right)+\right.\right.$

$$
\begin{aligned}
+\left[\widetilde{R}_{1(k)}^{[2]} \cdot \Phi_{1(k)}^{[2]}\left(\xi_{2}\right)+\right. & \left.\left.\left.\widetilde{R}_{2(k)}^{[2]} \cdot \Phi_{2(k)}^{[2]}\left(\xi_{2}\right)\right] \cdot C_{k}^{[1] "}\left(\xi_{1}\right)\right\}+\frac{a_{2}^{2}}{8 a_{1}^{2}}\left(\xi_{2}^{2}-1\right) 2 \cdot \frac{1}{a_{1}^{2}}\right] ; \\
& \frac{\partial^{2} w}{\partial x_{2}^{2}}=\left(\frac{q_{0} a_{1}^{4}}{D}\right)\left[\frac { 1 } { a _ { 2 } ^ { 2 } } \sum _ { k = 1 } ^ { K } \left\{\left[\widetilde{R}_{1(k)}^{[1]} \cdot \Phi_{1(k)}^{[1]}\left(\xi_{1}\right)+\widetilde{R}_{2(k)}^{[1]} \cdot \Phi_{2(k)}^{[1]}\left(\xi_{1}\right)\right] \cdot C_{k}^{[2] "}\left(\xi_{2}\right)+\right.\right. \\
& \left.\left.+\left[\widetilde{R}_{1(k)}^{[2]} \cdot \Phi_{1(k)}^{[2] "}\left(\xi_{2}\right)+\widetilde{R}_{2(k)}^{[2]} \cdot \Phi_{2(k)}^{[2]}\left(\xi_{2}\right)\right] \cdot C_{k}^{[1]}\left(\xi_{1}\right)\right\}+\frac{a_{2}^{2}}{8 a_{1}^{2}}\left(\xi_{1}^{2}-1\right) 2 \cdot \frac{1}{a_{2}^{2}}\right] ;
\end{aligned}
$$

Де

$$
\begin{aligned}
\Phi_{1(k)}^{[j, "}\left(\xi_{j}\right)= & \frac{1}{2} \exp \left[-\delta_{k} \cdot \frac{a_{j}}{a_{3-j}}\left(1-\xi_{j}\right)\right] \cdot\left(\delta_{k}\right)^{2} \cdot\left(\frac{a_{j}}{a_{3-j}}\right)^{2}+\frac{1}{2} \exp \left[-\delta_{k} \cdot \frac{a_{j}}{a_{3-j}}\left(1+\xi_{j}\right)\right]\left(\delta_{k}\right)^{2} \cdot\left(\frac{a_{j}}{a_{3-j}}\right)^{2} ; \\
& \left.\Phi_{2(k)}^{[j] \prime} \xi_{j}\right)=\exp \left[-\delta_{k} \cdot \frac{a_{j}}{a_{3-j}}\left(1-\xi_{j}\right)\right] \delta_{k} \cdot \frac{a_{j}}{a_{3-j}}+\frac{1}{2} \xi_{j} \cdot \exp \left[-\delta_{k} \cdot \frac{a_{j}}{a_{3-j}}\left(1-\xi_{j}\right)\right]\left(\delta_{k}\right)^{2} \cdot\left(\frac{a_{j}}{a_{3-j}}\right)^{2}+ \\
& +\exp \left[-\delta_{k} \cdot \frac{a_{j}}{a_{3-j}}\left(1+\xi_{j}\right)\right] \cdot \delta_{k} \cdot \frac{a_{j}}{a_{3-j}}-\frac{1}{2} \xi_{j} \exp \left[-\delta_{k} \cdot \frac{a_{j}}{a_{3-j}}\left(1+\xi_{j}\right)\right] \cdot\left(\delta_{k}\right)^{2} \cdot\left(\frac{a_{j}}{a_{3-j}}\right)^{2} ;
\end{aligned}
$$

проведені числові розрахунки розподілу прогину та згинних моментів в головних перерізах $\left(\xi_{j}=0\right)$ плити. Результат обчислень графічно представлені на рис. 1, 3, 5, 7, 9. 


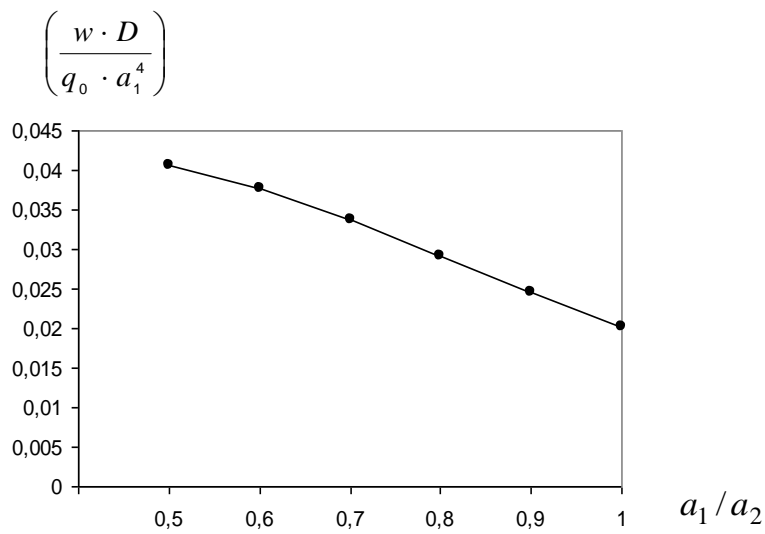

Рис. 1. Зміна максимального прогину плити в залежності від співвідношення іiї сторін.
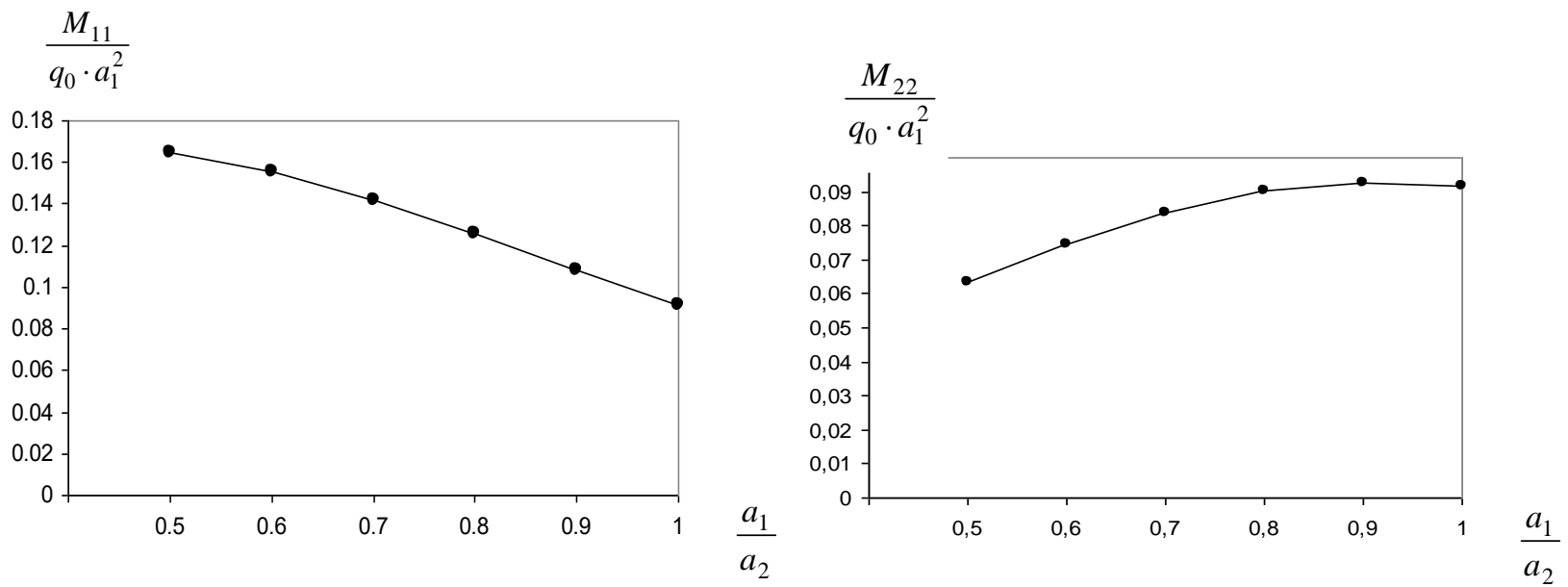

Рис. 2. Зміна максимального згинного моменту $M_{11} / q_{0} a_{1}^{2}$ в центрі плити в залежності від співвідношення іiі

сторін. Рис. 3. Зміна максимального згинного моменту $M_{22} / q_{0} a_{1}^{2}$ в центрі плити в залежності від співвідношення іiі сторін.
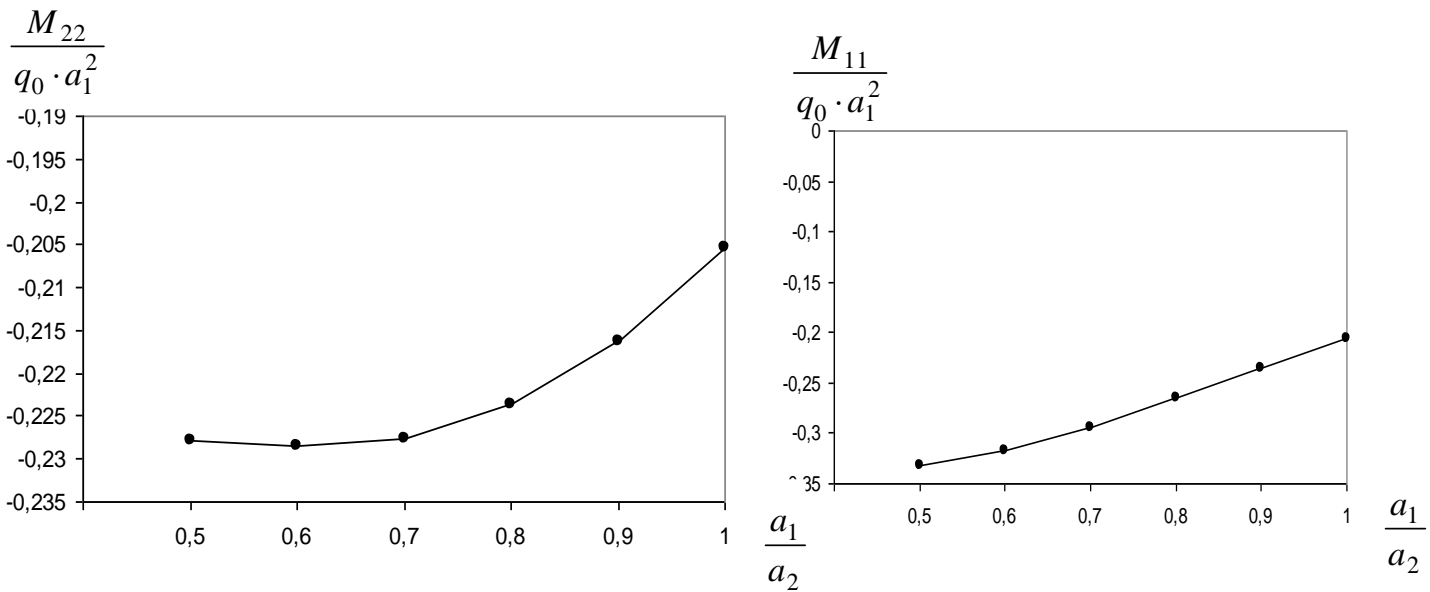

Рис. 4. Зміна максимального згинного моменту $M_{22} / q_{0} a_{1}^{2}$ на краю плити $\left(x_{2}=a_{2} ; x_{1}=0\right)$ в залежності від співвідношення іï сторін.Рис. 5. Зміна максимального згинного моменту $M_{11} / q_{0} a_{1}^{2}$ на краю плити $\left(x_{1}=a_{1} ; x_{2}=0\right)$ в залежності від співвідношення іiі сторін.На рисунках 1-5 представлені графіки зміни відносних величин прогину $w$, та згинних моментів $M_{11}, M_{22}$ в центрі плити та на їі краях в залежності від співвідношення сторін $a_{1} / a_{2}$. Залежність максимального прогину плити (в іiї центрі) від співвідношення $a_{1} / a_{2}$ ілюструє рисунок 1 . Із збільшенням $a_{1} / a_{2}$ прогин падає і досягає найменшого значення для квадратної плити. $\quad$ Графіки зміни моментів $M_{11}$ (рис.2) та $M_{22}$ (рис.3) в центрі плити мають протилежний характер: збільшення $a_{1} / a_{2}$ приводить до зростання моменту $M_{22}$ і падіння моменту 
$M_{11}$. Із збільшенням відношення $a_{1} / a_{2}$ згинний момент $M_{11}$ на краю плити $\left(x_{1}=a_{1}, x_{2}=a_{2}\right)$ суттєво зростає (рис.4) в той же час згинний момент $M_{22}$ (рис.5) майже не залежить від співвідношення сторін.

\section{Список бібліографічного опису.}

1. Тимошенко С.П., Войновски-Кригер. Пластины и оболочки.-М.: Наука, 1966.- 635c.

2. Бережницкий Л.Т., Делявский М.В.,Панасюк В.В. Изгиб тонких пластин с дефектами типа трещин.-Киев: Наукова думка, 1979.- 400с.

3. Прусов И.А. Метод сопряжения в теории плит.-Минск: Изд-во Белорус. ун-та, 1975.- 256 с.

4. Kączkowski Z. Płyty. Obliczenia statyczne.- Warszawa: Arkady, 1980.-

5. Делявский М.В. Расчет напряженного состояния в толстой ортотропной плите под действием изгибающей нагрузки № 11-12.- С. 117-123.

6. Delyavskyy M., Gołaś J., Podhorecka A.: O pewnym podejściu do rozwiązywania płyt wielowarstwowych, XLV Konf. Nauk. Krynica 99, s.63-70.

\section{References.}

1. Tymoshenko SP, Voynovsky-Krieger. Plates and casings. - M .: Nauka, 1966. - 635s.

2. Berezhnitsky LT, Delyavsky MV, Panasyuk VV Bending of thin plates with defects such as cracks. - Kiev: Naukova Dumka, 1979. - 400s.

3. Prusov IA The method of conjugation in the theory of plates.-Minsk: Publisher Belarus. Univ., 1975.- $256 \mathrm{p}$.

4. Kączkowski Z. Płyty. Obliczenia statyczne.- Warszawa: Arkady, 1980.-

5. Delyavsky MV Calculation of stress state in a thick orthotropic plate under the action of bending load N. 11-12.- P. 117123.

6. Delyavskyy M., Gołaś J., Podhorecka A.: On the Peculiar Adjournment to the Development of Pliyt Wielowarstwowych, XLV Conf. Nauk. Krynica 99, p.63-70. 